\title{
Cooling a charged mechanical resonator with time-dependent bias gate voltages
}

\author{
Jian-Qi Zhang ${ }^{1}$, Yong $\mathrm{Li}^{2,+}$ and Mang Feng ${ }^{1, *}$ \\ ${ }^{1}$ State Key Laboratory of Magnetic Resonance and Atomic and Molecular Physics, \\ Wuhan Institute of Physics and Mathematics, Chinese Academy of Sciences, Wuhan \\ 430071, China \\ 2 Beijing Computational Science Research Center, Beijing 100084, China \\ E-mail: ${ }^{+}$liyong@csrc.ac.cn, ${ }^{*}$ mangfeng@wipm.ac.cn
}

\begin{abstract}
We show a purely electronic cooling scheme to cool a charged mechanical resonator (MR) down to nearly the vibrational ground state by elaborately tuning bias gate voltages on the electrodes, which couple the MR by Coulomb interaction. The key step is the modification of time-dependent effective eigen-frequency of the MR based on the Lewis-Riesenfeld invariant. With respect to a relevant idea proposed previously [Li et al., Phys. Rev. A 83, 043803 (2011)], our scheme is simpler, more practical and completely within the reach of current technology.
\end{abstract}

PACS numbers: 07.20.Mc, 03.65.-w, 41.20.Cv 
Micro- and nano-mechanical resonators (MRs) have attracted much research interest due to their combination of both classical and quantum properties [1, 2] together with broad impact on fundamental researches and applications [3], such as ultra-sensitive measurements approaching quantum limit [3, 4], observation of continuous-variable entanglement with mesoscopic objects [5, 6], quantum information processing [7, and biological sensing [8].

The prerequisite of the research in these aspects is to cool the MRs down to their ground states in order to suppress detrimental influence from thermal fluctuations. Up to now, there have been many proposals for cooling the MRs $[9,10,11,12,13,14,15,16$, 17, 18, 19, 20, 21] in either optomechanical or electromechanical systems, in which the resolved sideband cooling method enables the vibrational ground-state cooling of MRs. So far, the ground-state cooling of MRs has been achieved experimentally [22, 23, 24, 25].

Very recently, a different approach to fast ground-state cooling of MRs has been proposed [16] with time-dependent optical driving in a three-mirror cavity optomechanical system, in which the effective frequency of the MR can be changed like an 'optical spring' and the MR can be cooled down to nearly its ground- state under the control of time-dependent external optical driving fields. But the scheme seems challenging experimentally due to the requirement for both adiabatic evolution under Born-Oppenheimer approximation and very strong optical powers.

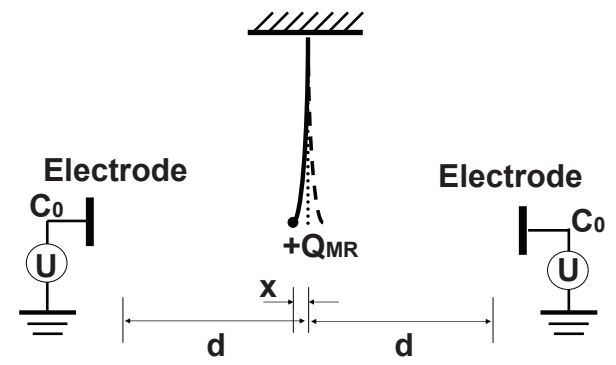

Figure 1. Schematic diagram of the system, where a charged MR with (positive) charge $Q_{M R}$ couples to two identical electrodes via Coulomb interaction. The two electrodes are distant by $2 d$ and $x$ is the deviation of the MR from the equilibrium position. $C_{0}$ is the capacitance of gate, and $U$ is the tunable bias gate voltage.

We present in this work an alternative method using a purely electronic way, which is simpler but more practical, for cooling a charged MR by two electrodes via Coulomb interaction. Under the control of bias gate voltages, the MR behaves as a single-mode harmonic oscillator with its effective frequency tunable like an 'electrical spring'. Our method is somewhat similar to that given in Ref. [16] but without involvement of Born-Oppenheimer approximation. Moreover, in contrast to Ref. [16], our idea is more feasible using current experimental techniques due to manipulation of bias gate voltages. Furthermore, different from the traditional cooling schemes in electromechanical systems based on superconductor qubits [17, 18] or microwave photons [11, 20], our scheme can cool a MR down to its vibrational ground state by simply tuning the bias gate voltages. To the best of our knowledge, this is the first cooling scheme with purely electronic 
way to cool the MR system without any assistance from auxiliary qubits or additional photons.

As schematically shown in Fig. 1, we consider a system where a charged MR is placed in the middle of two identical electrodes, and couples electrostatically to these two electrodes. Each electrode takes the charge $Q=C_{0} U$ with $C_{0}$ and $U$ being the capacitance and voltage of the bias gate, respectively. The Coulomb force between the $\mathrm{MR}$ and each electrode varies with the bias gate voltage, and the Coulomb potential is written by

$$
V_{c}=k C_{0} U Q_{M R}\left(\frac{1}{d+x}+\frac{1}{d-x}\right),
$$

where $d$ is the equilibrium separation between the MR center-of-mass position and the electrodes with $x$ the deviation of the MR from the equilibrium position, $k\left(=1 / 4 \pi \varepsilon_{0}\right)$ is the Coulomb constant in vacuum with $\varepsilon_{0}$ being the vacuum dielectric constant, and $Q_{M R}$ is the charge of the MR.

After defining $U=U_{0} f(t)$ with the dimensionless time-dependent factor $f(t)$ satisfying $|f(t)|<1$, we may reduce Eq. (1), under the condition of $d \gg x$, to

$$
V_{c} \simeq 2 k C_{0} U_{0} Q_{M R} f(t) x^{2} / d^{3} .
$$

where the term $2 k C_{0} U_{0} Q_{M R} f(t) / d$ has been ignored since it commutes with both the position and the momentum operators of the MR, and makes no contribution to following discussions.

Supposing the charged MR in the absence of the electrodes takes the Hamiltonian

$$
H_{m}=\frac{1}{2 m} p^{2}+\frac{1}{2} m \omega_{m}^{2} x^{2},
$$

where $x$ and $p$ are the position and momentum operators of the MR with the bare eigen-frequency $\omega_{m}$ and the effective mass $m$, we have the modified Hamiltonian under the control of the bias gate as

$$
\begin{aligned}
H & =H_{m}+V_{c} \\
& =\frac{1}{2 m} p^{2}+\frac{1}{2} m \omega_{\mathrm{eff}}^{2} x^{2}
\end{aligned}
$$

where the effective frequency is $\omega_{\text {eff }}=\omega_{m} \sqrt{1+\eta f(t)}$ with $\eta=4 k C_{0} U_{0} Q_{M R} /\left(m \omega_{m}^{2} d^{3}\right)$.

Generally speaking, the dynamics of a harmonic oscillator (e.g. MR) governed by Eq. (4) can be obtained by the method of Lewis-Riesenfeld invariants [27, 28, 26]. Particularly, for some special intermediate trajectories, the instantaneous quantum state of the harmonic oscillator at initial time instant can be the same as that at the final time instant, although the effective eigen-frequency of the harmonic oscillator has been changed significantly [28]. That is to say, we may have the final mean phonon number of the harmonic oscillator to be the same as the initial one, but with significant change of the effective temperature. This idea holds for arbitrary initial state, and has been used to cool the spatial motion of atoms [26] and to cool the MR in cavity optomechanical system [16].

Our present idea is to achieve cooling of the MR by modifying time-dependent eigen-frequency via tuning the bias gate voltages on external electrodes. We note that 
the effective frequency $\omega_{\text {eff }}$ of the MR can be easily changed by tuning the bias gate voltages $U$. In fact, $\omega_{\text {eff }}$ can be much smaller than $\omega_{m}$ under negative $U$. Moreover, $\omega_{\text {eff }}$ can be even imaginary in order to accelerate the cooling process [16, 26].

Specifically, considering some experimentally achievable parameters in charged MR systems [29, 32, 31, 30], such as $k=8.988 \times 10^{9} \mathrm{~N} \cdot \mathrm{m}^{2} / \mathrm{C}^{2}, \omega_{m}=2 \pi \times 134 \mathrm{kHz}, m=40$ $\mathrm{pg}, d=3.15 \mu \mathrm{m}, U_{0} \equiv 7.00 \mathrm{~V}, Q_{M R}=|e| \sigma_{M R} \times s, \sigma_{M R}=1.25 \times 10^{13} / \mathrm{cm}^{2}, C_{0}=27.5$ $\mathrm{nF}$, and $s=0.08 \mu \mathrm{m}^{2}$, we have $\eta \simeq 1.25 \times 10^{7}$, meeting the approximate condition in Eq. (2).

Similar to Ref. [16], our protocol for cooling MR comprises following two steps.

Step I: Decreasing the mean phonon number with the increase of the MR effective frequency

In the absence of bias gate voltages (i.e., $f\left(t_{o}\right)=0$ ), we assume the MR with eigenfrequency $\omega_{\text {eff }}\left(t_{o}\right)=\omega_{m}$ initially in a thermal equilibrium state

$$
\rho\left(t_{o}\right)=e^{-\frac{H\left(t_{o}\right)}{k_{B} T}} / \operatorname{Tr}\left(e^{-\frac{H\left(t_{o}\right)}{k_{B} T}}\right)
$$

at temperature $T=20 \mathrm{mK}$. So the corresponding mean thermal phonon number is given by

$$
\bar{n}\left(t_{o}\right)=1 /\left\{\exp \left[\hbar \omega_{\text {eff }}\left(t_{o}\right) / k_{B} T\right]-1\right\} \simeq 3100 .
$$

With an arbitrary trajectory of $\omega_{\text {eff }}(t)$ [or $\left.f(t)\right]$, the MR is assumed to be in a new thermal state

$$
\rho\left(t_{i}\right)=e^{-H\left(t_{i}\right) /\left(k_{B} T\right)} / \operatorname{Tr}\left[e^{-H\left(t_{i}\right) /\left(k_{B} T\right)}\right],
$$

at a later time $t_{i}$ with $f\left(t_{i}\right)=1$, where the effective frequency is $\omega_{\text {eff }}\left(t_{i}\right) \simeq 3500 \omega_{m}$ and the mean thermal phonon number is

$$
\bar{n}\left(t_{i}\right)=\frac{1}{\exp \left[\hbar \omega_{\text {eff }}\left(t_{i}\right) /\left(k_{B} T\right)\right]-1} \simeq 0.47 .
$$

It is clear that the temperature of the MR at the time $t_{i}$ remains unchanged compared with the initial one, while the mean thermal phonon number of the MR is much less than the one at the initial time $t_{o}$ due to enlargement of the effective frequency $\omega_{\text {eff }}$. The fact $\bar{n}\left(t_{i}\right) \simeq 0.47<1$ means that the MR with enlarged effective frequency has been cooled down to nearly the vibrational ground state.

Step II: Decreasing the MR effective frequency by keeping the low mean phonon number

This step is accomplished via a special trajectory of $f(t)$ evolving to the final time $t_{f}$ under the control of bias gate voltages satisfying two conditions: i) The final effective frequency is equal to the bare one (i.e., $\left.\omega_{\text {eff }}\left(t_{f}\right)=\omega_{m}\right)$, implying that the bias gate voltages are absent again at the final time $t_{f}$ (i.e., $f\left(t_{f}\right)=0$ ); ii) During the cooling process, the mean phonon number remains unchanged compared with that at time $t_{i}$ 
(i.e., $\bar{n}\left(t_{f}\right)=\bar{n}\left(t_{i}\right) \simeq 0.47$ ). Consequently, we have the MR with the bare frequency cooled to nearly the vibrational ground state.

The solution to such a special trajectory of $f(t)$ between $t_{i}$ and $t_{f}$ governed by Eq. (44) follows the Lewis-Riesenfeld invariant [27, 28, 26]. Using the inverse-invariant method [28, 33] in Appendix, we obtain the control function of the trajectory of $f(t)$ by tracing the Lewis-Riesenfeld invariant,

$$
f(t)=\frac{\omega_{\text {eff }}^{2}(t)-\omega_{m}^{2}}{\eta \omega_{m}^{2}}=\frac{\omega_{0}^{2}-b(t)^{3} \ddot{b}(t)-\omega_{m}^{2} b(t)^{4}}{\eta b(t)^{4} \omega_{m}^{2}},
$$

where the dimensionless function $b(t)$ is defined in Appendix.

The trajectory of $f(t)$, starting from $f\left(t_{i}=0\right)=1$ and ending with $f\left(t_{f}\right)=0$, corresponds to the effective frequency of the MR decreasing from the end frequency in the first step $\left(\omega_{\text {eff }}\left(t_{i}\right) \equiv \omega_{0} \simeq 3500 \omega_{m}\right)$ to the final frequency $\omega_{\text {eff }}\left(t_{f}\right)=\omega_{m}$. We plot in Fig. 2 the control function $f(t)$, the corresponding instantaneous eigen-frequency $\omega_{\text {eff }}(t)$ and the effective temperature $T_{\text {eff }}$.
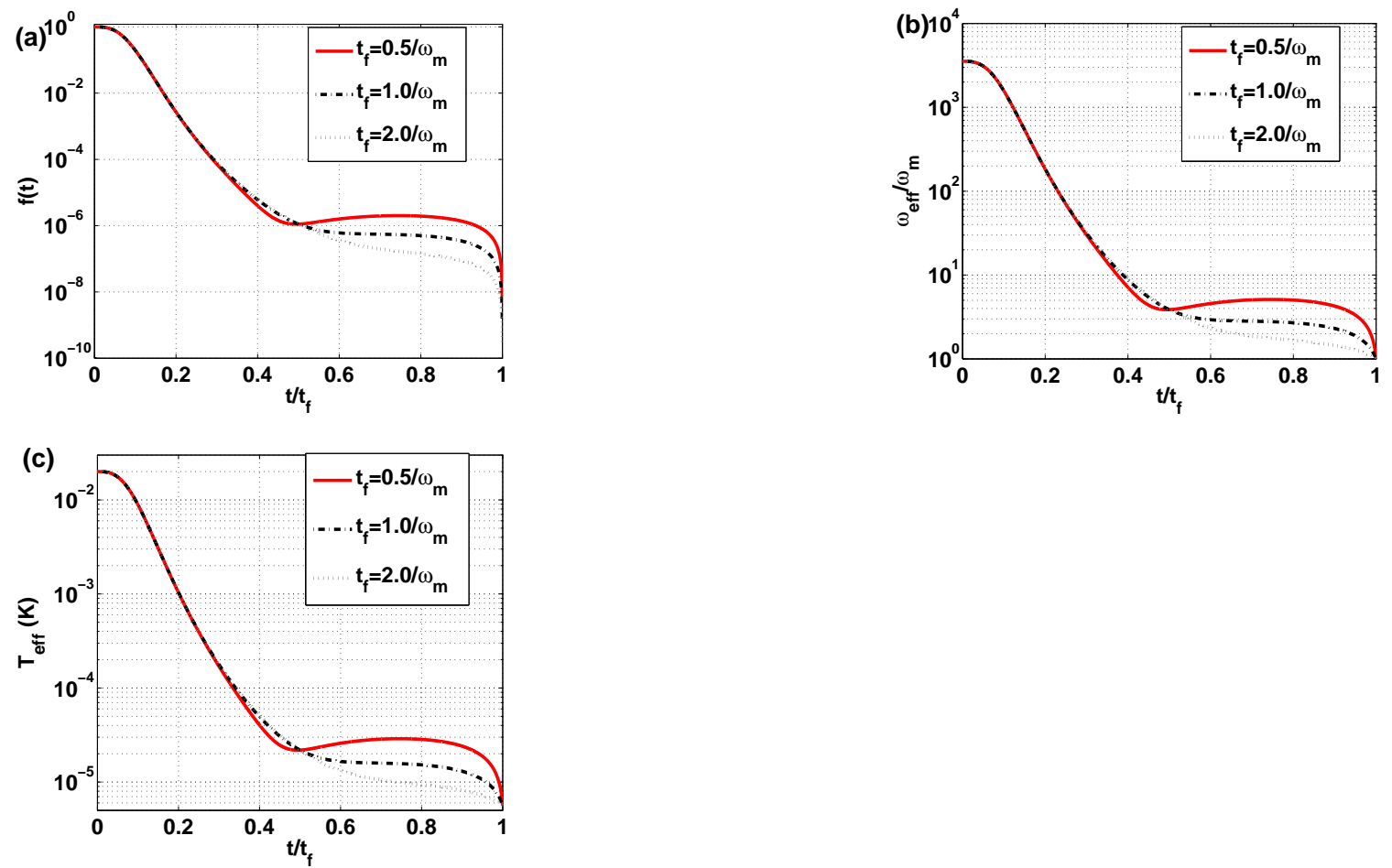

Figure 2. (Color online) (a) Time evolution of $f(t)$; (b) Time evolution of the corresponding effective frequency $\omega_{\text {eff }}$; (c) Time evolution of the effective temperature of the MR. In each panel, we consider three cases: $t_{f}=0.5 / \omega_{m}$ (red solid line), $t_{f}=1.0 / \omega_{m}$ (black dotted-dashed line), and $t_{f}=2.0 / \omega_{m}$ (gray dotted line). The parameter values are taken as $\omega_{m}=2 \pi \times 134 \mathrm{kHz} ; m=40 \mathrm{pg} ; \sigma_{M R}=1.25 \times 10^{13} / \mathrm{cm}^{2}$, $C_{0}=27.52 \mathrm{nF}, d=3.15 \mu \mathrm{m}$, and $s=0.04 \mu \mathrm{m}^{2}$ from [29, 32, 31, 30].

Using Eq. (5), we know that our cooling enables the MR from the initial temperature $T_{\text {eff }}\left(t_{i}\right)=T_{\text {eff }}\left(t_{o}\right)=T=20 \mathrm{mK}$ down to the final temperature $T_{\text {eff }}\left(t_{f}\right)=6 \mu \mathrm{K}$, 

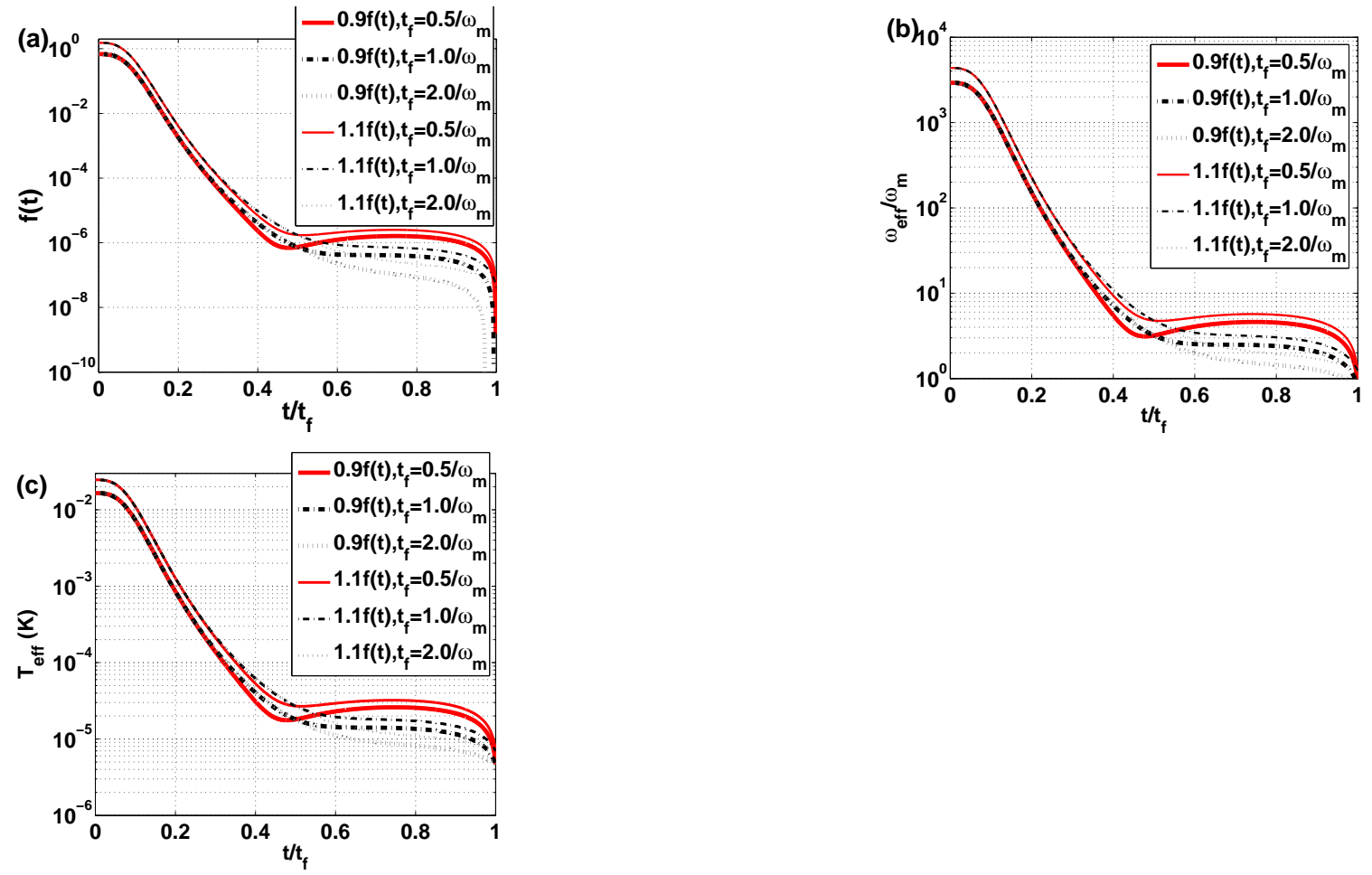

Figure 3. (Color online) (a) Time evolution of $f(t)$ with $10 \%$ error (i.e., $0.9 f(t)=$ $(1-10 \%) f(t)$ and $1.1 f(t)=(1+10 \%) f(t))$; (b) Time evolution of the effective frequency $\omega_{\text {eff } f}$ under the different trajectory of $\mathrm{f}(\mathrm{t})$; (c) Time evolution of the effective temperature of the MR under the different trajectory of $\mathrm{f}(\mathrm{t})$. In each plot, we consider two kinds: $0.9 f(t)=(1-10 \%) f(t)$ (thick line), and $0.9 f(t)=(1-10 \%) f(t)$ (thin line). Except $f(t)$, other parameters are the same as in Fig 2.

as plotted in Fig. 2(c). Here the effective temperature is defined through $\bar{n}(t) \equiv$ $1 /\left\{\exp \left[\hbar \omega_{e f f}(t) / k_{B} T_{\text {eff }}(t)\right]-1\right\}$.

To estimate the imperfection in the trajectory of $f(t)$, which corresponds to the experimental error in tunable bias gate voltages on electrodes, we give the trajectory of $f(t)$ a ten percent fluctuation, i.e., $(1 \pm 10 \%) f(t)$, as in Fig. 3(a), and simulate in Fig. 3(b) and (c) the changes of the instantaneous eigen-frequency $\omega_{\text {eff }}(t)$ of the MR and the corresponding effective temperature of the MR. We find that, although the experimental deviation is as large as $10 \%$, the effective eigen-frequency can still reach $1.23 \omega_{m}$ for $+10 \% f(t)\left(0.84 \omega_{m}\right.$ for $\left.-10 \% f(t)\right)$ deviation with the corresponding effective temperature $7 \mu \mathrm{K}$ for $+10 \% f(t)(5 \mu \mathrm{K}$ for $-10 \% f(t))$ deviation. In other words, the MR in such cases can still be cooled down to nearly its vibrational ground state. Moreover, we note that the final effective temperature $5 \mu \mathrm{K}$ for $-10 \% f(t)$ deviation (See Fig. 33(c)) seems lower than $6 \mu \mathrm{K}$ under the ideal condition. But this is the case of fluctuation, which is uncontrollable. In addition, in this deviating case, the effective frequency is $0.83 \omega_{m}$, rather than the desired effective frequency $\omega_{m}$. Therefore, our choice of trajectory in Fig. 3 (b) is definitely optimal.

We would like to point out that our scheme is only for an instantaneous cooling, 
which is different from the usual sideband cooling scheme achievable in steady state. This is because the MR is decoupled from electrodes at the end of our cooling process. So the MR cannot be kept in low temperature for very long time but be heated again by environment.

However, after the cooling, the MR would not return to the bath temperature suddenly due to its high $\mathrm{Q}$ factor $\left(>10^{5}\right)$ [22, 23, 24, 25] which makes sure the small decay of the MR. As a result, there should be a long enough time to finish the scheduled experimental works, such as quantum information processing [7] before the MR is heated to the bath temperature. Even if the MR is thermalized to the bath temperature, we may repeat the same cooling process to put the MR down to the vibrational ground state again. Therefore, both the cooling process and the experimental work should be done alternately in the implementation of the scheduled experimental works. This is similar to that in ion trap system, wherein one carries out the sideband cooling and the operations of quantum algorithms alternately [34].

In summary, we have proposed a practical protocol for cooling a MR near to its vibrational ground state by controlling bias gate voltages on two nearby electrodes. Using the achievable experimental parameters and considering experimental imperfection, we have shown the feasibility of the proposal by currently available technology. As a result, our work is not only a practical cooling scheme, but also very promising to verify the Lewis-Resisenfeld invariant method experimentally.

\section{Appendix}

We present below how to obtain the special trajectory of $f(t)$ between $t=t_{i}(=0)$ and $t=t_{f}$ by means of the Lewis-Riesenfeld invariant. Based on the inverse-invariant method [28, 33], Eq. (44) satisfies the time-dependent invariant [27],

$$
I=\frac{1}{2}\left[\frac{x^{2}}{b(t)^{2}} m \omega_{0}^{2}+\frac{\Lambda^{2}}{m}\right],
$$

where $\omega_{0}=\omega_{\text {eff }}\left(t_{i}\right)$ for simplicity, $\Lambda \equiv b(t) p-m \dot{b}(t) x$ takes the role of the momentum conjugate to $x / b(t)$, and the dimensionless real function $b(t)$ follows

$$
\ddot{b}(t)+\omega_{\text {eff }}^{2}(t) b(t)=\frac{\omega_{0}^{2}}{b(t)^{3}} .
$$

According to the boundary conditions at time $t_{i}$ and the finial time $t_{f}$, we have

$$
\begin{aligned}
& b\left(t_{i}\right)=1, \quad \dot{b}\left(t_{i}\right)=0, \quad \ddot{b}\left(t_{i}\right)=0, \\
& b\left(t_{f}\right)=\chi, \quad \dot{b}\left(t_{f}\right)=0, \quad \ddot{b}\left(t_{f}\right)=0,
\end{aligned}
$$

with $\chi=\sqrt{\omega_{0} / \omega_{\text {eff }}\left(t_{f}\right)}$. So the simplest polynomial $b(t)$ between $t_{i}(=0)$ and $t_{f}$ is given by

$$
b(t)=6(\chi-1) s^{5}-15(\chi-1) s^{4}+10(\chi-1) s^{3}+1
$$

with $s=t / t_{f}$. 


\section{Acknowledgments}

The work is supported by the National Fundamental Research Program of China (Grant Nos. 2012CB922102, 2012CB922104, 2009CB929604 and 2007CB925204) and the National Natural Science Foundation of China (Grant Nos. 10974225, 60978009 and 11174027).

[1] Braginsky V B and Manukin A B Measurements of Weak Forces in Physics Experiments, edited by Douglass D H (Chicago: Chicago University Press) 1977, p.161.

[2] Wei L F, Liu Y X, Sun C P and Nori F, 2006 Phys. Rev. Lett. 97237201.

[3] Marquardt F and Girvin S M, 2009 Physics 240.

[4] Forstner S, Prams S, Knittel J, van Ooijen E D, Swaim J D, Harris G I, Szorkovszky A, Bowen W P and Rubinsztein-Dunlop H, 2012 Phys. Rev. Lett. 108120801.

[5] Tan H T and Li G X, 2011 Phys. Rev. A 84024301.

[6] Hartmann M J and Plenio M B, 2008 Phys. Rev. Lett. 101200503.

[7] Stannigel K, Rabl P, Sorensen A S, Zoller P and Lukin M D, 2010 Phys. Rev. Lett. 105220501.

[8] Tetard L, Passian A, Venma K T.r, Lynch R M, Voy B H, G Shekhawat, V P Dravid and T Thundat, 2008 Nat. Nanotechnol. 3501.

[9] Groblacher S, Hammerer K, Vanner M and Aspelmeyer M, 2009 Nature (London) 460724.

[10] Thompson J D, Zwickl B M, Jayich A M, Marquardt F, Girvin S M and Harris J G E, 2008 Nature (London) 45272.

[11] Li Y, Wang Y D, Xue F and Bruder C, 2008 Phys. Rev. B 78134301.

[12] Wilson-Rae I, Zoller P and Imamoglu A, 2004 Phys. Rev. Lett. 92075507.

[13] Wilson-Rae I, Nooshi N, Zwerger W and Kippenberg T J, 2007 Phys. Rev. Lett. 99093901.

[14] Marquardt F, Chen J P, Clerk A A and Girvin S M, 2007 Phys. Rev. Lett. 99093902.

[15] Xia K and Evers J, Phys. Rev. Lett. 1032009227203.

[16] Li Y, Wu L A and Wang Z D, Phys. Rev. A 832011043804.

[17] Li Y, Wu L A, Wang Y D and Yang L P, 2011 Phys. Rev. B 84094502.

[18] Wang Y D, Li Y, Xue F, Bruder C, and Semba K, 2009 Phys. Rev. B 80144508.

[19] Mari A and Eisert J, 2012 Phys. Rev. Lett. 108120602.

[20] Xue F, Wang Y D, Liu Y X and Nori F, 2007 Phys. Rev. B 76205302.

[21] Metzger C H and Karrai K, 2004 Nature (London) 4321002.

[22] O'Connell A D, et al., 2010 Nature (London) 464697.

[23] Chan J, Alegre T P M, Safavi-Naeini A H, Hill J T, Krause A, Groblacher S, Aspelmeyer M and Painter O, 2011 Nature (London) 47889.

[24] Groblacher S, Hertzberg J B, Vanner M R, Cole G D, Gigan S, Schwab K C and Aspelmeyer M, 2009 Nat. Phys. 5485.

[25] Rocheleau T, Ndukum T, Macklin C, Hertzberg J B, Clerk A A and Schwab K C, 2010 Nature (London) 46372.

[26] Chen X, Ruschhaupt A, Schmidt S, del Campo A, Guery-Odelin D and Muga J G, 2010 Phys. Rev. Lett. 104063002.

[27] Lewis H R and Riesenfeld W B, 1969 J Math Phys 101458.

[28] Muga J G, Chen X, Ibanez S, Lizuain I and Ruschhaupt A, 2010 J. Phys. B 4385509.

[29] Thompson J D, B M Zwickl, A M Jayich, F Marquardt, S M Girvin and J G E Harris, 2008 Nature (London) 45272.

[30] LaHaye M D, Buu O, Camarota B and Schwab K, 2004 Science 30474.

[31] Hensinger W K, Utami D W, Goan H S, Schwab K, Monroe C and Milburn G J, 2005 Phys. Rev. A 72041405 .

[32] Kong Y C, Zheng Y D, Zhou C H, Deng Y Z, Gu S L, Shen B, Zhang R, Han P, Jiang R L and Shi Y, 2004 Acta Phys Sin 532324.

[33] Palao J P, Muga J G and Sala R, 1998 Phys. Rev. Lett. 805469. 
[34] Roos C F, 2000 PhD Thesis: Controlling the quantum state of trapped ions (Innsbruck University). 